\title{
Dynamic Congested Traffic States of Density Difference Lattice Hydrodynamic Model with On-Ramp
}

\author{
Jun-fang Tian, Zhen-zhou Yuan, Bin Jia, and Wang Tao \\ MOE Key Laboratory for Urban Transportation Complex Systems Theory and Technology, Beijing Jiaotong University, \\ Beijing 100044, China \\ Correspondence should be addressed to Jun-fang Tian; tianhustbjtu@hotmail.com
}

Received 1 June 2013; Revised 21 August 2013; Accepted 23 August 2013

Academic Editor: Tetsuji Tokihiro

Copyright (C) 2013 Jun-fang Tian et al. This is an open access article distributed under the Creative Commons Attribution License, which permits unrestricted use, distribution, and reproduction in any medium, provided the original work is properly cited.

\begin{abstract}
The density difference lattice hydrodynamic model with on-ramp is proposed. Stochastic and deterministic rules for the on-ramp flow entering into the main road are designed. Under the stochastic rule, various empirical spatiotemporal patterns can be well reproduced, such as the pinned localized cluster (PLC) and homogeneous synchronized traffic (HST). Under the deterministic rule, four new types of congested traffic states, such as the stationary oscillatory congested traffic (OCT) upstream of on-ramp and the stationary and localized OCT emerging in HST, are identified for the first time. Comparisons with the macroscopic speed gradient model are carried out, and some advantages of our model are clarified. To our knowledge, it is the first research showing that the lattice hydrodynamic model could reproduce various congested patterns induced by the on-ramp system.
\end{abstract}

\section{Introduction}

With the rapid development of urbanization, traffic congestion becomes one of the most serious problems that undermine the operation efficiency of modern cities. In order to understand the mechanism of traffic congestion, many models and analyses have been carried out to explain the empirical findings [1-7], such as the hysteresis, stability, and phase transitions. Usually, there are two types of traffic models, that is, microscopic and macroscopic models. Microscopic models, including cellular automata [8] and carfollowing models [9-11], focus on the dynamics of individual vehicles. Macroscopic models, including gas-kinetic-based models [12], continuum models $[13,14]$ and lattice hydrodynamic models [15-29], regard the whole traffic flow as a flow of continuous medium composed of vehicles. Individual vehicles do not appear explicitly in macroscopic models.

Recently, phase transitions induced by the on-ramp have been observed and studied intensively. Helbing et al. [3036] found that traffic states in the open systems with bottlenecks are mainly determined by the instability diagram. The preconditions for the possible occurrence of different traffic states can be illustrated by the phase diagram. These empirical traffic states include the pinned localized cluster (PLC), moving localized cluster (MLC), triggered stop-and-go traffic (TSG), oscillating congested traffic (OCT), homogeneous synchronized traffic (HST), and homogeneous congested traffic (HCT); see Figure 1.

In previous works, both microscopic and macroscopic models except the lattice hydrodynamic models were investigated under the on-ramp system. The lattice hydrodynamic models were firstly proposed by Nagatani $[15,16]$ and later extended by many other researchers [17-29], due to their simple structure, ease of theoretical analysis and simulation, and deep connection with the macroscopic continuum models and microscopic car-following models. Maybe the lacking of the lattice hydrodynamic model with on-ramp is the reason why the phase transitions and congested states induced by the on-ramp have never been investigated by these models.

In this paper, we firstly propose the density difference lattice hydrodynamic model with on-ramp. Later two types of on-ramp system are designed. We show that not only all observed congested traffic states can be simulated by the model mentioned before but also some new congested states are found. The remainder of the present paper is organized as follows. In the following section, DDLM is introduced. 


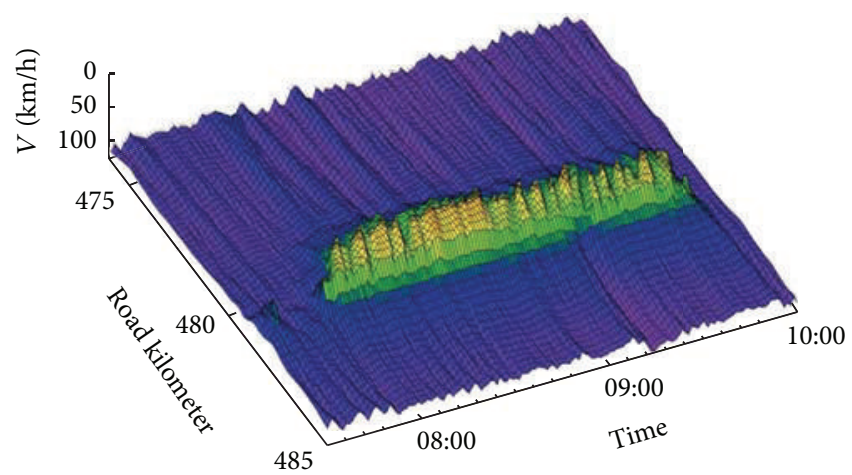

(a) Pinned localized cluster (PLC) A5 South, September 19, 2001

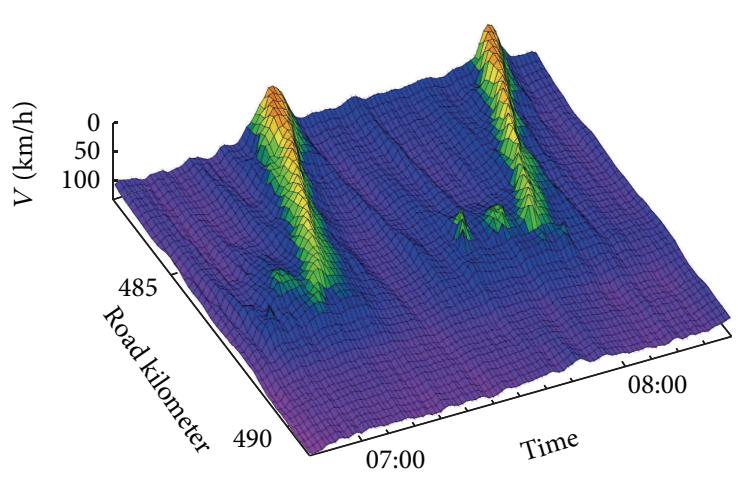

(b) Moving localized cluster (MLC) A5 South, June 06, 2001

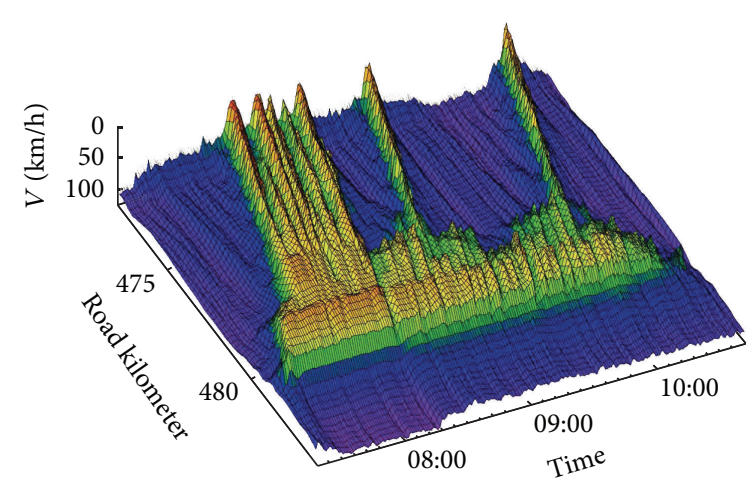

(c) Triggered stop-and-go waves (TSG) A5 South, May 07, 2001

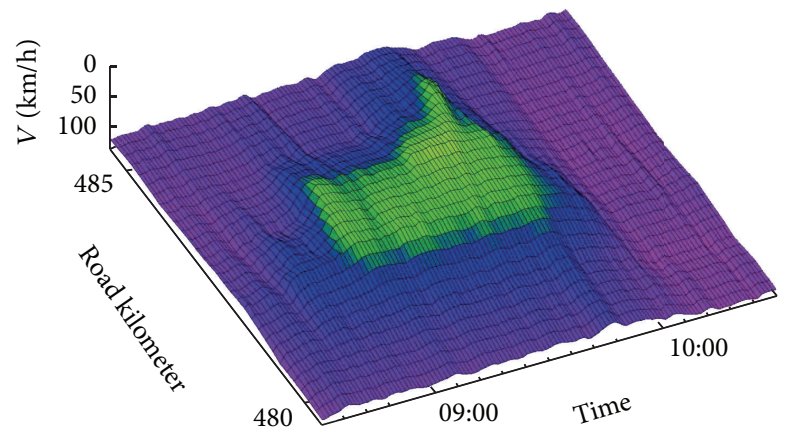

(e) Homogeneous synchronized traffic (HST) A5 South, April 09, 2001

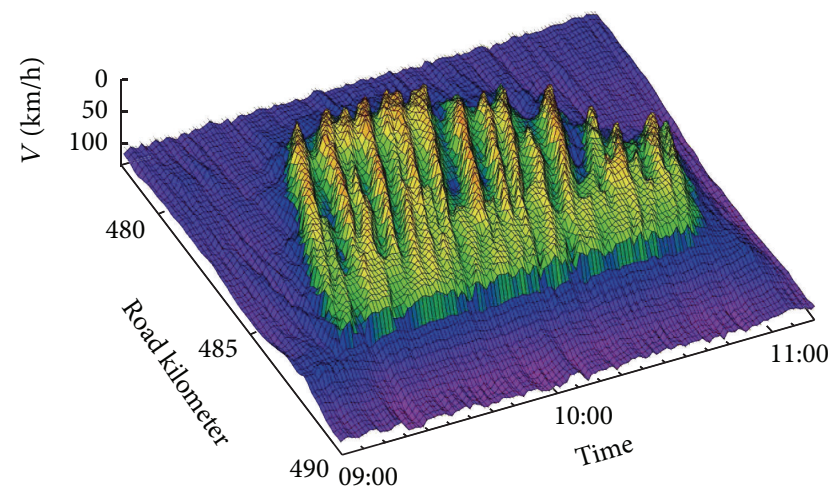

(d) Oscillating congested traffic (OCT) A5 South, July 31, 2001

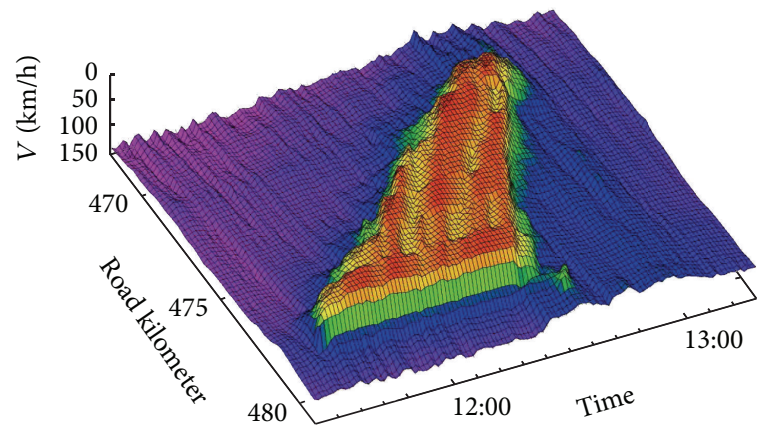

(f) Homogenous congested traffic (HCT) A5 South, April 25, 2001

Figure 1: Examples of congested traffic states measured on the German freeway A5 close to Frankfurt. (a) Pinned localized cluster (PLC), (b) Moving localized cluster (MLC), (c) Triggered stop-and-go waves (TSG), (d) Oscillating congested traffic (OCT), (e) Homogeneous synchronized flow (HST), and (f) Homogeneous congested traffic (HCT). This figure is taken from http://www.traffic-states.com/.

In Section 3, DDLM with on-ramp is proposed and the onramp systems for lattice hydrodynamic models are designed. In Section 4, numerical simulations are carried out. Finally, conclusions are given in Section 5.

\section{Density Difference Lattice Hydrodynamic Model}

In 1998, Nagatani $[15,16]$ proposed the first lattice hydrodynamic model. Nagatani's model was derived by discretizing the following continuum model:

$$
\begin{gathered}
\partial_{t} \rho+\partial_{x}(\rho v)=0, \\
\partial_{t}(\rho v)=a \rho_{0} V(\rho(x+\delta))-a \rho v,
\end{gathered}
$$

where $a$ is the sensitivity of the driver and $\delta$ represents the average headway, $\delta=1 / \rho_{0}$. The $\rho(x+\delta)$ is the local density at position $x+\delta$ at time $t$, which is related to the inverse of headway $h(x, t)$; that is, $\rho(x+\delta)=1 / h(x, t) . V(\rho(x+\delta))$ is the optimal velocity.

In Nagatani's original paper $[15,16], \rho_{0}$ is defined as the average density. Then (2) can describe correctly steady-state traffic flow (subscript $e$ ): $\rho_{0}=\rho, \rho(x+\delta)=\rho(x)$, and 
$d(\rho v) / d t(\rho v)=0$, so $v_{e}(\rho)=V(\rho)$ which is consistent. However, in this interpretation, a definition of the averaging procedure to obtain $\rho_{0}$ from $\rho$ has not been presented. Moreover the real traffic system is an open system, which leads to difficulties formulating this procedure. Therefore, we think $\rho_{0}$ should be considered as a constant parameter. Although it makes the steady-state relationship not consistent since from (2) it follows that $v_{e}(\rho)=\rho_{0} / \rho V(\rho)$ which diverges for $\rho$ to zero rather than tending to $V(0)$, but it is definite that the traffic is in the very low density free flow state if $\rho$ is smaller than $\rho$ due to the small value of $\rho_{0}$. We think this state is not the focus of traffic flow, which is the congested traffic. Thus, this deficiency will have little influence on the application of the model.

The idea of this model is that a driver adjusts the vehicle velocity according to the observed headway $h(x, t)$ or density ahead $\rho(x+\delta)$. This is similar to the idea in the carfollowing models with the optimal velocity $V(h(x, t))$. Then, for dimensionless space $x$ (let $\tilde{x}=x / \delta$, and $\tilde{x}$ is indicated as $x$ hereafter), the lattice model is expressed as:

$$
\begin{gathered}
\partial_{t} \rho_{j}+\rho_{0}\left(\rho_{j} v_{j}-\rho_{j-1} v_{j-1}\right)=0, \\
\partial_{t}\left(\rho_{j} v_{j}\right)=a \rho_{0} V\left(\rho_{j+1}\right)-a \rho_{j} v_{j},
\end{gathered}
$$

where $j$ denotes the site on the one-dimensional lattice and $\rho_{j}$ and $v_{j}$ denote the local density and the local average velocity on site $j$ at time $t$, respectively.

Subsequently, many extended models [17-29] have been proposed to suppress traffic jams. In particular, the density difference lattice hydrodynamic model (DDLM) [28, 29], by incorporating the density difference ahead into Nagatani's model, shows a close relationship with the gas-kinetic-based model $[30,31]$ and the information that density difference plays a significant role in traffic flow. DDLM consists of continuity equation (4) and dynamic equation (5):

$$
\begin{gathered}
\partial_{t} \rho_{j}+\rho_{0}\left(\rho_{j} v_{j}-\rho_{j-1} v_{j-1}\right)=0 \\
\partial_{t}\left(\rho_{j} v_{j}\right)=a \rho_{0} V\left(\rho_{j+1}\right)-a \rho_{j} v_{j}+\frac{\lambda\left(\rho_{j}-\rho_{j+1}\right)}{\rho_{0}},
\end{gathered}
$$

where $\lambda$ is the reaction coefficient to the density difference. By eliminating $v$ in (4) and (5), the following equation is obtained:

$$
\begin{aligned}
\partial_{t}^{2} \rho_{j} & +a \rho_{0}^{2}\left(V\left(\rho_{j+1}\right)-V\left(\rho_{j}\right)\right)+a \partial_{t} \rho_{j} \\
& +\lambda\left(2 \rho_{j}-\rho_{j+1}-\rho_{j-1}\right)=0
\end{aligned}
$$

One can see that DDLM has considered the following lattice $j-1$. For the convenience of simulation, we rewrite (6) into the difference form:

$$
\begin{aligned}
\rho_{j}(t+2 \tau)= & 2 \rho_{j}(t+\tau)-\rho_{j}(t) \\
& -a \tau^{2} \rho_{0}^{2}\left(V\left(\rho_{j+1}(t)\right)-V\left(\rho_{j}(t)\right)\right) \\
& -a \tau\left(\rho_{j}(t+\tau)-\rho_{j}(t)\right) \\
& -\lambda \tau^{2}\left(2 \rho_{j}(t)-\rho_{j+1}(t)-\rho_{j-1}(t)\right) .
\end{aligned}
$$

The optimal velocity is chosen as follows [16]:

$$
V(\rho)=\frac{v_{\max }}{2}\left[\tanh \left(\frac{2}{\rho_{0}}-\frac{\rho}{\rho_{0}^{2}}-\frac{1}{\rho_{c}}\right)+\tanh \left(\frac{1}{\rho_{c}}\right)\right],
$$

where $v_{\max }$ is the maximum speed and $\rho_{c}$ is the critical density. This function has the turning point at $\rho=\rho_{0}=$ $\rho_{c}$. Typical values of these parameters are $\rho_{c}=\rho_{0}=$ 0.25 and $v_{\max }=2 \mathrm{~m} / \mathrm{s}[15,16]$. Figure 2 is the schematic illustration of the speed and flow as a function of the density. According to the maximum value of flow on the flow-density curve, free traffic and congested traffic can be classified, which is applied to distinguish the traffic state in the following simulations.

\section{Density Difference Lattice Hydrodynamic Model with On-Ramp}

In previous studies [15-29], theoretical analysis and numerical simulations for lattice hydrodynamic models focus on suppressing traffic jams and linear and nonlinear stability analysis. Traffic states induced by the on-ramp systems have not been investigated.

In order to simulate the empirical phenomena observed by Helbing et al. [30-36], two simple methods are designed to model the on-ramp system for the lattice model. In Figure 3(a), the position of the on-ramp is located at $x_{\text {on }}$ and the region $\left[x_{\mathrm{on}}, x_{\mathrm{on}}+L_{\mathrm{ramp}}\right]$ is selected as the inserting area of the flux from on-ramp, where $L_{\text {ramp }}$ is the length of the on-ramp. At each time step, a lattice $s$ in this region will be selected randomly. However, the length of the inserting area is set as $L_{\text {ramp }}=1$ in Figure 3(b); that is, the flux from on-ramp could enter the main road by only one lattice. According to the different inserting rules of the on-ramp flux, Figures 3(a) and 3(b) are simply named as the "stochastic on-ramp" and "deterministic on-ramp," respectively.

The on-ramp flux $Q_{\text {on }}(t)$ will be inserted into the selected lattice. The density difference lattice model with the on-ramp could be described as follows:

$$
\begin{gathered}
\partial_{t} \rho_{j}+\rho_{0}\left(\rho_{j} v_{j}-\rho_{j-1} v_{j-1}\right)=\rho_{0} \bar{Q}_{j}, \\
\partial_{t}\left(\rho_{j} v_{j}\right)=a \rho_{0} V\left(\rho_{j+1}\right)-a \rho_{j} v_{j}+\frac{\lambda\left(\rho_{j}-\rho_{j+1}\right)}{\rho_{0}},
\end{gathered}
$$

where

$$
\bar{Q}_{j}(t)= \begin{cases}Q_{\text {on }}(t): & \text { if } j=s, \\ 0: & \text { if } j \neq s .\end{cases}
$$




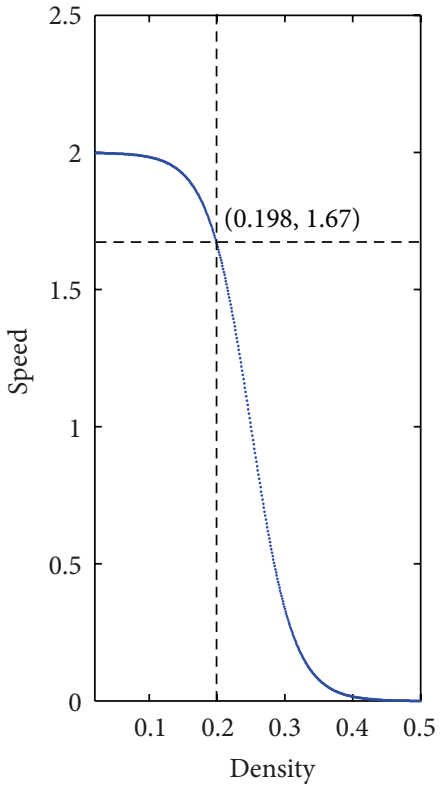

(a)

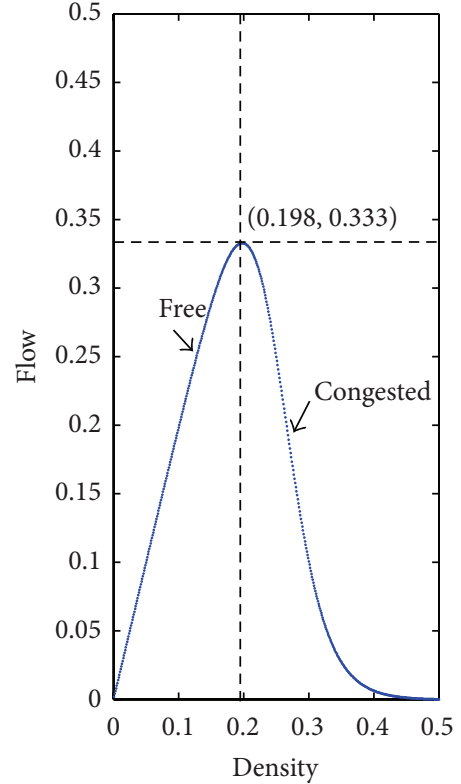

(b)

FIGURE 2: Schematic illustration of speed and flow as a function of the density for $\rho_{c}=\rho_{0}=0.25, v_{\max }=2 \mathrm{~m} / \mathrm{s}$. (a) The speed-density curve. (b) The flow-density curve.

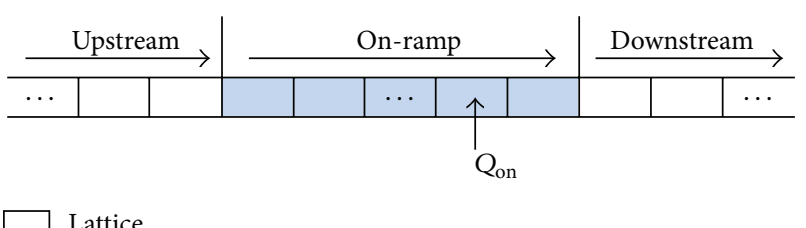

(a)

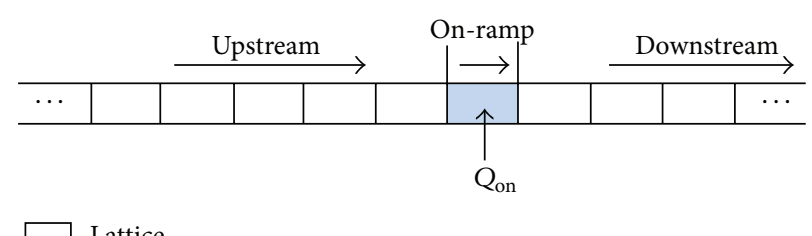

(b)

Figure 3: The sketches of the on-ramp systems. (a) The stochastic on-ramp, (b) the deterministic on-ramp.

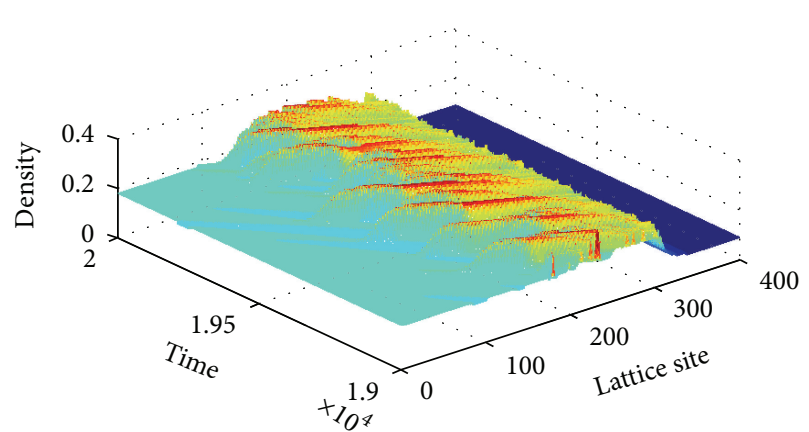

(a)

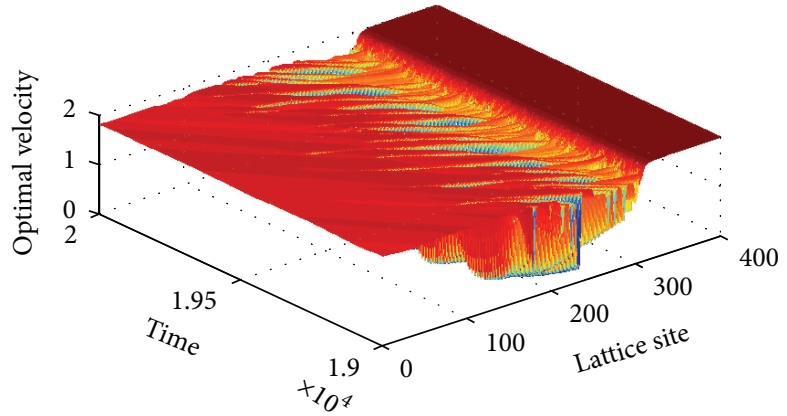

(b)

FIgUre 4: The spatiotemporal evolutions of PLC for $\rho_{h}=0.08, Q_{\text {on }}=0.13$.

By eliminating $v$ in (9) and writing the results into the difference form, the following model is obtained:

$$
\begin{aligned}
\rho_{j}(t+2 \tau)= & 2 \rho_{j}(t+\tau)-\rho_{j}(t) \\
& -a \tau^{2} \rho_{0}^{2}\left(V\left(\rho_{j+1}(t)\right)-V\left(\rho_{j}(t)\right)\right)
\end{aligned}
$$

$$
\begin{aligned}
& -a \tau\left(\rho_{j}(t+\tau)-\rho_{j}(t)\right) \\
& -\lambda \tau^{2}\left(2 \rho_{j}(t)-\rho_{j+1}(t)-\rho_{j-1}(t)\right) \\
& +a \tau^{2} \rho_{0} Q_{j}(t) .
\end{aligned}
$$




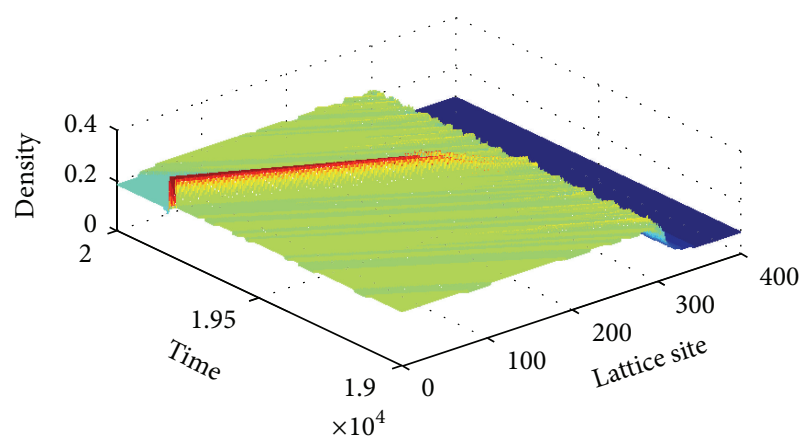

(a)

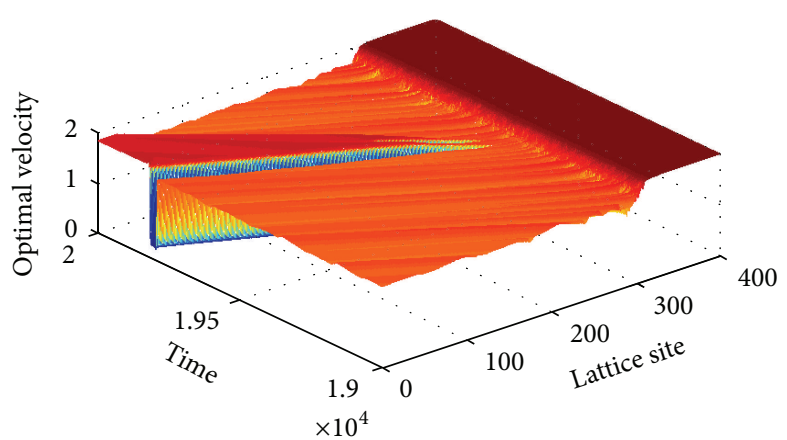

(b)

FIgURE 5: The spatiotemporal evolutions of MLC for $\rho_{h}=0.05, Q_{\text {on }}=0.1$.

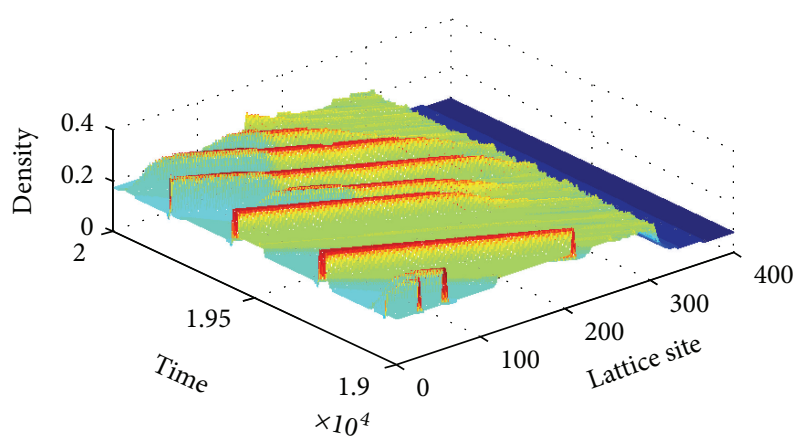

(a)

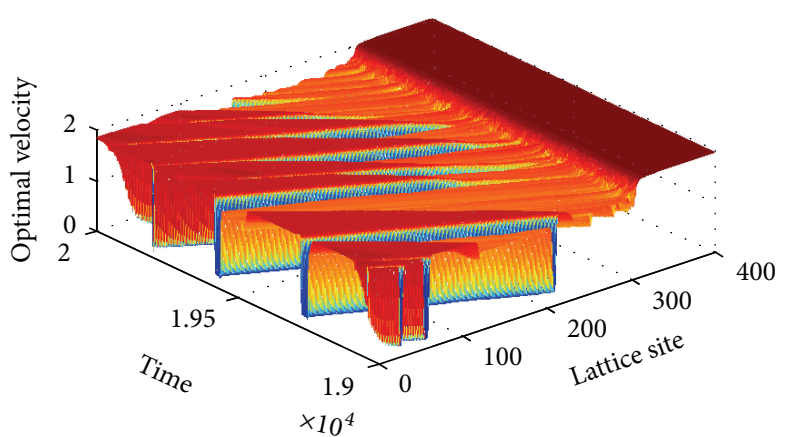

(b)

FIgURE 6: The spatiotemporal evolutions of TSG for $\rho_{h}=0.07, Q_{\mathrm{on}}=0.11$.

\section{Numerical Simulations}

The initial conditions for numerical simulations are set as follows. The main road is composed by $N=400$ lattices and the open boundary condition is applied; that is,

$$
\rho_{1}(t)=\rho_{2}(t), \quad \rho_{N-1}(t)=\rho_{N}(t) .
$$

The main road is prepared to start with a homogeneous traffic flow, whose density is $\rho_{h}$. The on-ramp region is located at $x_{\text {on }}=300$. The length of the stochastic on-ramp is $L_{\text {ramp }}=$ 10 lattices. The sensitivity $a$ is set as a piecewise constant function: $a=0.5$ for $\rho_{j} \leq 0.19$ and $a=1$ for $\rho_{j}>0.19$. Other parameters are set as follows: $\tau=0.1, \rho_{c}=\rho_{0}=0.25, v_{\max }=$ $2 \mathrm{~m} / \mathrm{s}$, and $\lambda=0.2$.

4.1. Stochastic On-Ramp Simulation. In Figure 4, the congested traffic is localized at the on-ramp, which is characterized by the localized reduction of speed and increase of density. Thus, it is the pinned localized cluster (PLC). The downstream front of the PLC is fixed at the bottleneck and its upstream front does not propagate upstream continuously over time. Upstream and downstream PLC, there is free traffic flow. Figure 5 shows the moving localized cluster (MLC). The traffic is congested with a single density wave. This density wave is a compression wave and propagates upstream with a constant speed. In contrast to the PLC, both the downstream and upstream fronts of MLC propagate on the road. Figure 6 shows the triggered stop-and-go traffic (TSG). Compared with MLC, there are several moving clusters on the road. Figure 7 exhibits the oscillatory congested traffic (OCT). Density waves emerge at the downstream and propagate upstream continuously. Figure 8 shows the homogeneous synchronized traffic (HST). The downstream front of HST is fixed at the downstream boundary and the upstream front of HST propagates upstream continuously over time. The density profile in Figure 9 is homogeneous over space except for the neighborhood of the downstream boundary. It is the homogeneous congested state (HCT). Differently from HST, vehicles' speed is very low due to the high density in HCT.

Furthermore, it should be noted that the congested clusters simulated by DDLM emerge in the congested traffic flow rather than free traffic flow. Comparing with Figure 1, one can see that the empirical congested traffic states can be well reproduced by DDLM.

4.2. Deterministic On-Ramp Simulation. In this section, we investigate the congested traffic states induced by the deterministic on-ramp. We found that HST and HCT can be reproduced, but the PLC, TSG, and MLC cannot be simulated. Moreover, the following new types of congested traffic states have been found.

Figure 10 shows the moving congested states. Its upstream front is composed by several clusters, that is, MLCs, and there are two clusters in Figure 10. Oscillations make up 


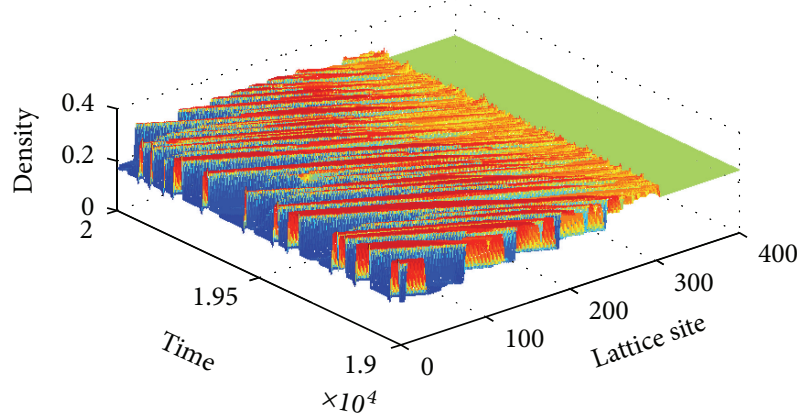

(a)

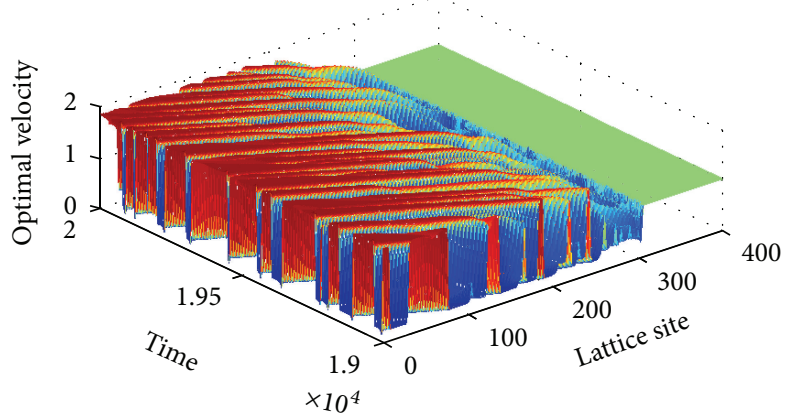

(b)

Figure 7: The spatiotemporal evolutions of OCT for $\rho_{h}=0.25, Q_{\mathrm{on}}=0.12$.

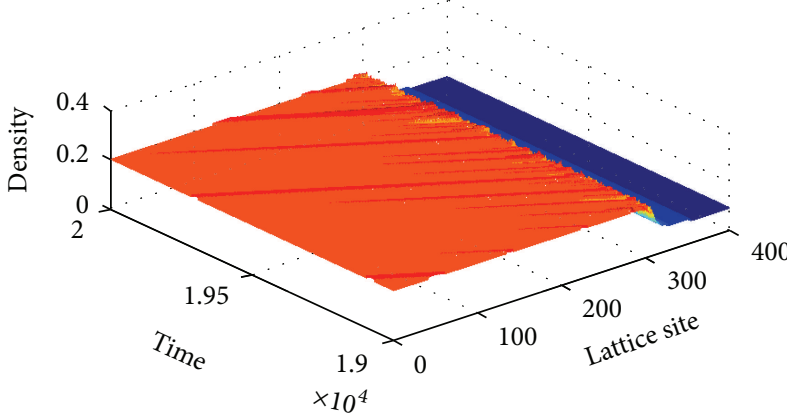

(a)

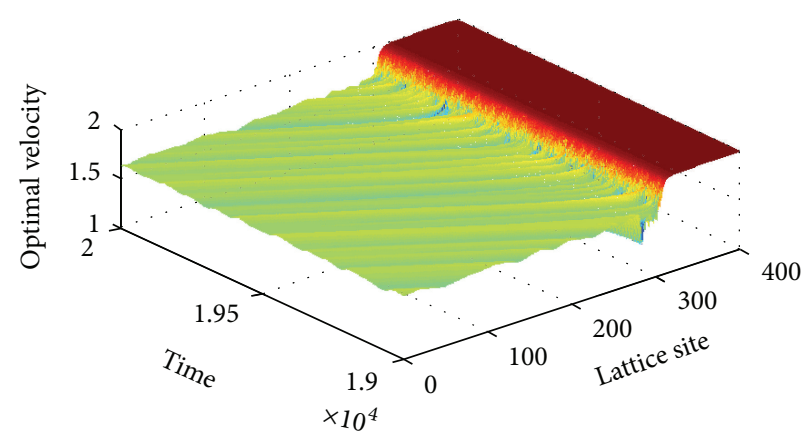

(b)

FIgURE 8: The spatiotemporal evolutions of HST for $\rho_{h}=0.09, Q_{\text {on }}=0.09$.

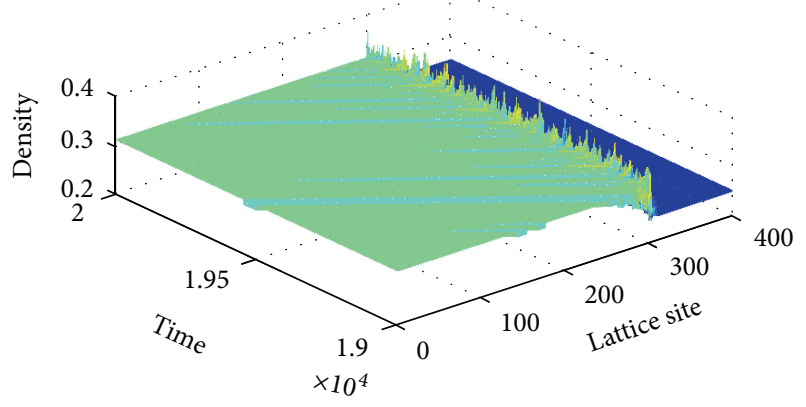

(a)

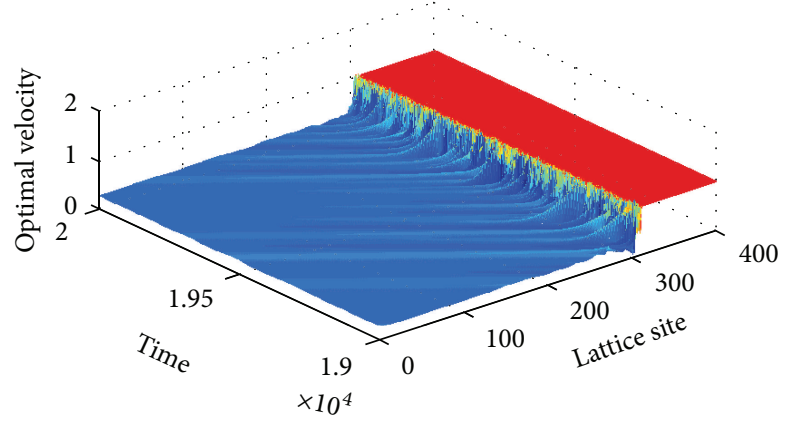

(b)

FIGURE 9: The spatiotemporal evolutions of HCT for $\rho_{h}=0.25, Q_{\text {on }}=0.18$.

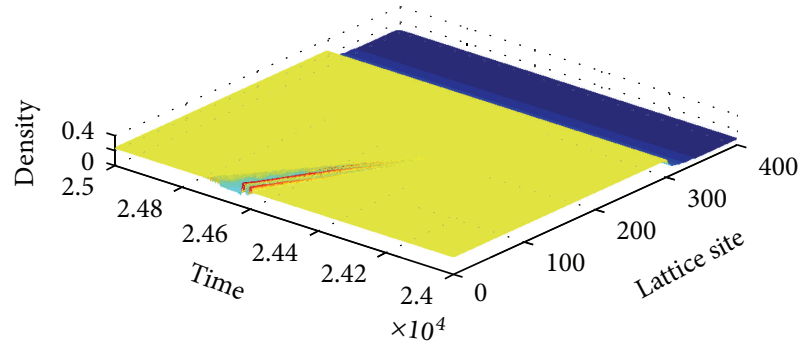

(a)

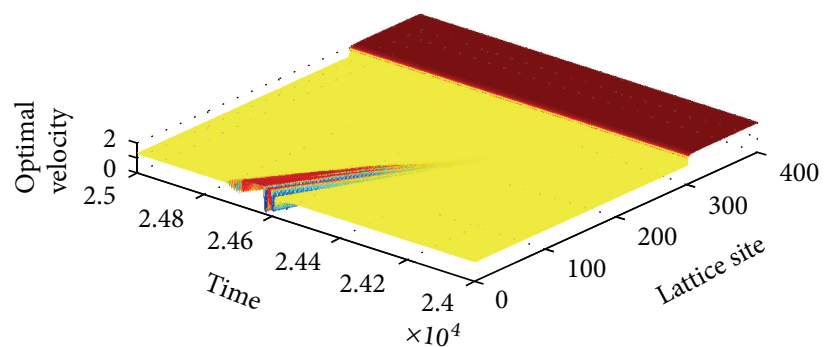

(b)

FIgURE 10: The spatiotemporal evolutions for $\rho_{h}=0.05, Q_{\mathrm{on}}=0.15$. 


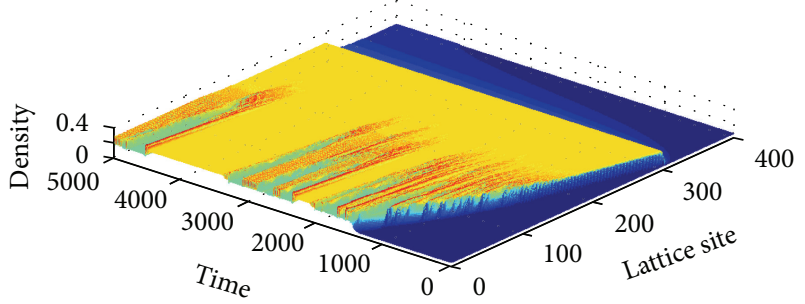

(a)

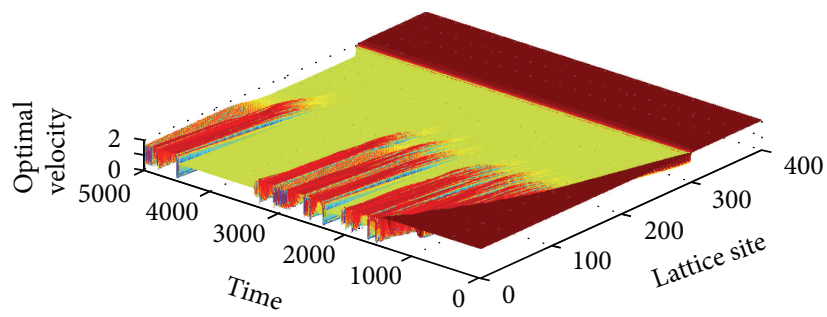

(b)

FIgURE 11: The spatiotemporal evolutions for $\rho_{h}=0.05, Q_{\text {on }}=0.18$.

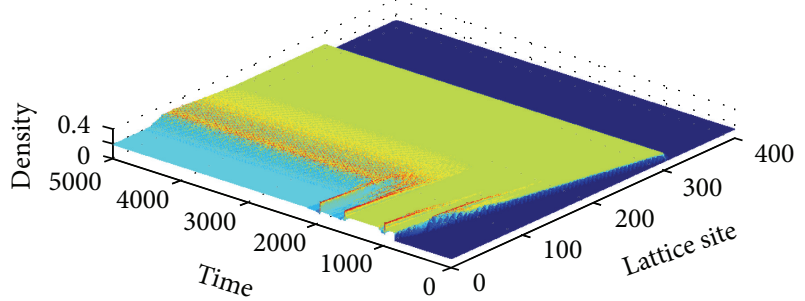

(a)

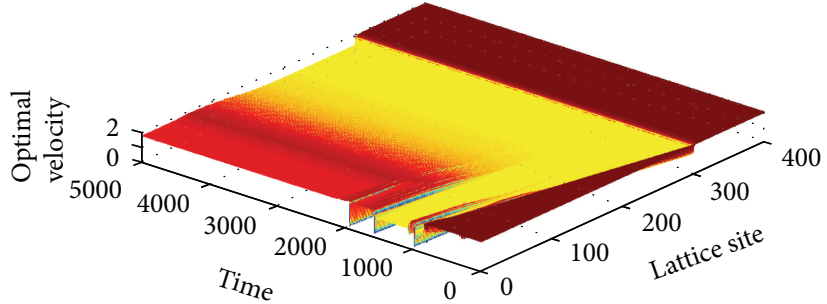

(b)

FIGURE 12: The spatiotemporal evolutions for $\rho_{h}=0.12, Q_{\text {on }}=0.14$

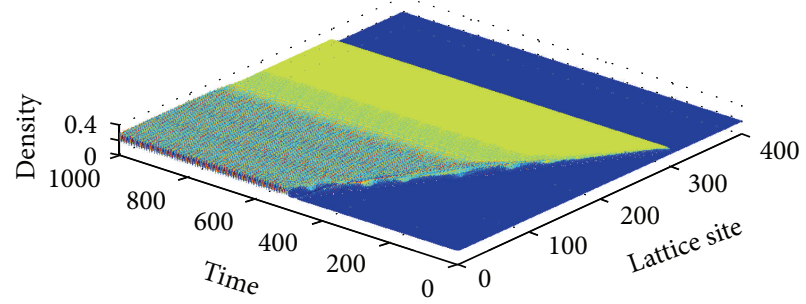

(a)

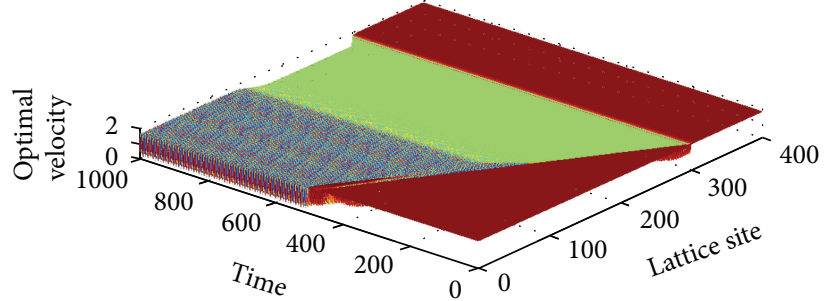

(b)

FIgURE 13: The spatiotemporal evolutions for $\rho_{h}=0.18, Q_{\mathrm{on}}=0.18$.

its downstream front, that is, moving OCT. Free flow exits between MLCs and OCT. In the other regions of the road, the HST is maintained. Thus, MLCs, OCT, free flow, and HST coexist in this congested state. Figure 11 shows another type of moving congested state. MLCs firstly occur in HST in this state. Then, free flow appears in the downstream of MCLs. Later, the moving OCT emerges in free flow. Usually, several regions of moving OCT and free flow alternately arise in this congested state. Figure 12 shows the free flow, OCT. and HST coexistence state. Before this state is formed, MLCs occur in HST. Then the OCT emerges far from the on-ramp. In the downstream front of OCT, HST is maintained. In the upstream front of OCT, free flow is preserved. The OCT is stationary and localized on the road. Figure 13 shows the HST and OCT coexistence state. The downstream front of OCT is away from the on-ramp; that is, this OCT is a stationary OCT. Downstream of the front, HST is maintained.

Moreover, we compare these results with those of other models, such as the macroscopic speed gradient model (SGM) [37]. The following conclusions can be made. Firstly, all empirical congested states can be simulated by both models. However, the simulated states of DDLM are more in accordance with the empirical data, such as the simulated PLC; TSG by SGM is only composed by two wave fronts. No congested regions exist between these fronts, which is inconsistent with the empirical PLC and TSG (see Figure 1). Secondly, the congested states (Figures 10-12) of DDLM induced by the deterministic on-ramp cannot be reproduced by SGM. Thirdly, similar state of Figure 13 is reproduced by SGM, which is made up by the stationary OCT and HCT.

\section{Conclusions}

Congested traffic states induced by the on-ramp have been observed and investigated widely by the macroscopic and microscopic traffic flow models. However, these have never been studied by the lattice hydrodynamic models.

In this paper, we have proposed the density difference lattice hydrodynamic model with on-ramp, in order to study the congested traffic states induced by the on-ramp. Stochastic and deterministic on-ramps are designed. Under the stochastic on-ramp, various empirical congested states 
are reproduced, such as the pinned localized cluster (PLC), moving localized cluster (MLC), triggered stop-and-go traffic (TSG), oscillating congested traffic (OCT), homogeneous synchronized traffic (HST), and homogeneous congested traffic (HCT). Under the deterministic on-ramp, four new types of congested traffic states are identified, such as the moving congested states whose upstream and downstream fronts are composed by MLCs and moving OCT, respectively, the moving congested state composed by several regions of moving OCT and free flow, the congested state that the free flow, the stationary and localized OCT and HST coexistence state, and the HST and OCT coexistence state. It is the first time that these new congested states have been found. Empirical data should be explored to demonstrate whether they exist in the real traffic. At last, comparisons with the macroscopic speed gradient model are carried out. We found that the simulation results of DDLM are more in accordance with the real traffic.

At last, it should be noted that the essential difference between the stochastic and deterministic on-ramps is the form of perturbations imposed on the main road. According to results in Section 4, we can conclude that different form of perturbations, not the amplitudes of perturbations, can lead to different types of congested traffic states. Thus, we infer that the empirical findings of three-phase theory may be reproduced if a suitable form of perturbations is applied.

\section{Acknowledgments}

The authors sincerely thank the referees and the editor for their comments on the paper. The authors wish to thank http://www.traffic-states.com/ for the empirical congested traffic states. This work is supported by the Fundamental Research Funds for the Central Universities (2013YJS052) and the 973 Program (no. 2012CB725403).

\section{References}

[1] D. Helbing, "Traffic and related self-driven many-particle systems," Reviews of Modern Physics, vol. 73, no. 4, pp. 1067-1141, 2001.

[2] T. Nagatani, "The physics of traffic jams," Reports on Progress in Physics, vol. 65, no. 9, p. 1331, 2002.

[3] K. Nagel, P. Wagner, and R. Woesler, "Still flowing: approaches to traffic flow and traffic jam modeling," Operations Research, vol. 51, no. 5, pp. 681-710, 2003.

[4] B. S. Kerner, The Physics of Traffic: Empirical Freeway Pattern Features, Engineering Applications, and Theory, Springer, 2004.

[5] B. Jia, Z. Y. Gao, K. P. Li, and X. G. Li, Models and Simulations of Traffic System Based on the Theory of Cellular Automaton, Science Press, Beijing, China, 2007.

[6] B. S. Kerner, Introduction to Modern Traffic Flow Theory and Control: The Long Road to Three-Phase Traffic Theory, Springer, 2009.

[7] M. Treiber and A. Kesting, Traffic Flow Dynamics: Data, Models and Simulation, Springer, Heidelberg, Germany, 2013.

[8] K. Nagel and M. Schreckenberg, "A cellular automaton model for freeway traffic," Journal de Physique I, vol. 2, no. 12, pp. 22212229, 1992.
[9] M. Bando, K. Hasebe, A. Nakayama, A. Shibata, and Y. Sugiyama, "Dynamical model of traffic congestion and numerical simulation," Physical Review E, vol. 51, no. 2, pp. 1035-1042, 1995.

[10] R. Jiang, Q. Wu, and Z. Zhu, "Full velocity difference model for a car-following theory," Physical Review E, vol. 64, no. 1, Article ID 017101, 2001.

[11] M. Treiber, A. Hennecke, and D. Helbing, "Congested traffic states in empirical observations and microscopic simulations," Physical Review E, vol. 62, no. 2, pp. 1805-1824, 2000.

[12] D. Helbing, A. Hennecke, V. Shvetsov, and M. Treiber, "MASTER: macroscopic traffic simulation based on a gas-kinetic, non-local traffic model," Transportation Research B, vol. 35, no. 2, pp. 183-211, 2001.

[13] R. Jiang, Q.-S. Wu, and Z.-J. Zhu, "A new continuum model for traffic flow and numerical tests," Transportation Research B, vol. 36, no. 5, pp. 405-419, 2002.

[14] T.-Q. Tang, H.-J. Huang, and Z.-Y. Gao, "Stability of the carfollowing model on two lanes," Physical Review E, vol. 72, no. 6, Article ID 066124, 7 pages, 2005.

[15] T. Nagatani, "Modified KdV equation for jamming transition in the continuum models of traffic," Physica A, vol. 261, no. 3-4, pp. 599-607, 1998.

[16] T. Nagatani, "TDGL and MKdV equations for jamming transition in the lattice models of traffic," Physica A, vol. 264, no. 3-4, pp. 581-592, 1999.

[17] H. X. Ge, S. Q. Dai, Y. Xue, and L. Y. Dong, "Stabilization analysis and modified Korteweg-de Vries equation in a cooperative driving system," Physical Review E, vol. 71, no. 6, Article ID 066119, 7 pages, 2005.

[18] H. X. Ge and R.-J. Cheng, "The "backward looking" effect in the lattice hydrodynamic model," Physica A, vol. 387, no. 28, pp. 6952-6958, 2008.

[19] H. X. Ge, R. J. Cheng, and L. Lei, "The theoretical analysis of the lattice hydrodynamic models for traffic flow theory," Physica A, vol. 389, no. 14, pp. 2825-2834, 2010.

[20] Y. Xue, "Lattice models of the optimal traffic current," Acta Physica Sinica, vol. 53, no. 1, pp. 25-30, 2004.

[21] T.-Q. Tang, H.-J. Huang, and Y. Xue, "An improved two-lane traffic flow lattice model," Acta Physica Sinica, vol. 55, no. 8, pp. 4026-4031, 2006.

[22] W.-X. Zhu and E.-X. Chi, "Analysis of generalized optimal current lattice model for traffic flow," International Journal of Modern Physics C, vol. 19, no. 5, pp. 727-739, 2008.

[23] J.-F. Tian, B. Jia, X.-G. Li, and Z.-Y. Gao, "Flow difference effect in the lattice hydrodynamic model," Chinese Physics B, vol. 19, no. 4, Article ID 040303, 2010.

[24] G. H. Peng, X. H. Cai, B. F. Cao, and C. Q. Liu, "Non-lanebased lattice hydrodynamic model of traffic flow considering the lateral effects of the lane width," Physics Letters A, vol. 375, no. 30-31, pp. 2823-2827, 2011.

[25] G. H. Peng, X. H. Cai, C. Q. Liu, and B. F. Cao, "A new lattice model of traffic flow with the consideration of the driver's forecast effects," Physics Letters A, vol. 375, no. 22, pp. 2153-2157, 2011.

[26] T. Wang, Z.-Y. Gao, X.-M. Zhao, J.-F. Tian, and W.-Y. Zhang, "Flow difference effect in the two-lane lattice hydrodynamic model," Chinese Physics B, vol. 21, no. 7, Article ID 070507, 2012.

[27] W.-X. Zhu and L.-D. Zhang, "A novel lattice traffic flow model and its solitary density waves," International Journal of Modern Physics C, vol. 23, no. 3, Article ID 1250025, 2012. 
[28] J.-F. Tian, Z. Yuan, B. Jia, M.-H. Li, and G.-J. Jiang, “The stabilization effect of the density difference in the modified lattice hydrodynamic model of traffic flow," Physica A, vol. 391, no. 19, pp. 4476-4482, 2012.

[29] J.-F. Tian, Z.-Z. Yuan, B. Jia, and H.-Q. Fan, "Phase transitions and the Korteweg-de Vries equation in the density difference lattice hydrodynamic model of traffic flow," International Journal of Modern Physics C, vol. 24, no. 3, Article ID 1350016, 9 pages, 2013.

[30] D. Helbing and M. Treiber, "Gas-kinetic-based traffic model explaining observed hysteretic phase transition," Physical Review Letters, vol. 81, no. 14, pp. 3042-3045, 1998.

[31] D. Helbing and M. Treiber, "Numerical simulation of macroscopic traffic equations," Computing in Science and Engineering, vol. 1, no. 5, pp. 89-98, 1999.

[32] H. Y. Lee, H.-W. Lee, and D. Kim, "Dynamic states of a continuum traffic equation with on-ramp," Physical Review E, vol. 59, no. 5, pp. 5101-5111, 1999.

[33] H. Y. Lee, H. W. Lee, and D. Kim, "Phase diagram of congested traffic flow: an empirical study," Physical Review E, vol. 62, no. 4, pp. 4737-4741, 2000.

[34] D. Helbing, A. Hennecke, and M. Treiber, "Phase diagram of traffic states in the presence of inhomogeneities," Physical Review Letters, vol. 82, no. 21, pp. 4360-4363, 1999.

[35] M. Schonhof and D. Helbing, "Criticism of three-phase traffic theory," Transportation Research B, vol. 43, no. 7, pp. 784-797, 2009.

[36] M. Treiber, A. Kesting, and D. Helbing, "Three-phase traffic theory and two-phase models with a fundamental diagram in the light of empirical stylized facts," Transportation Research $B$, vol. 44, no. 8-9, pp. 983-1000, 2010.

[37] C.-F. Tang, R. Jiang, and Q.-S. Wu, "Phase diagram of speed gradient model with an on-ramp," Physica A, vol. 377, no. 2, pp. 641-650, 2007. 


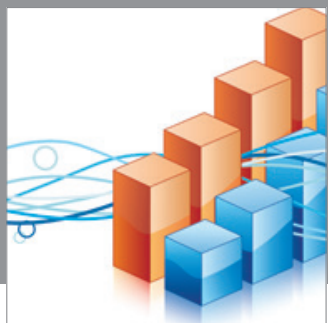

Advances in

Operations Research

mansans

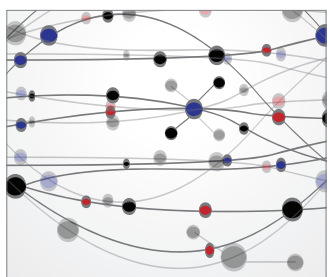

The Scientific World Journal
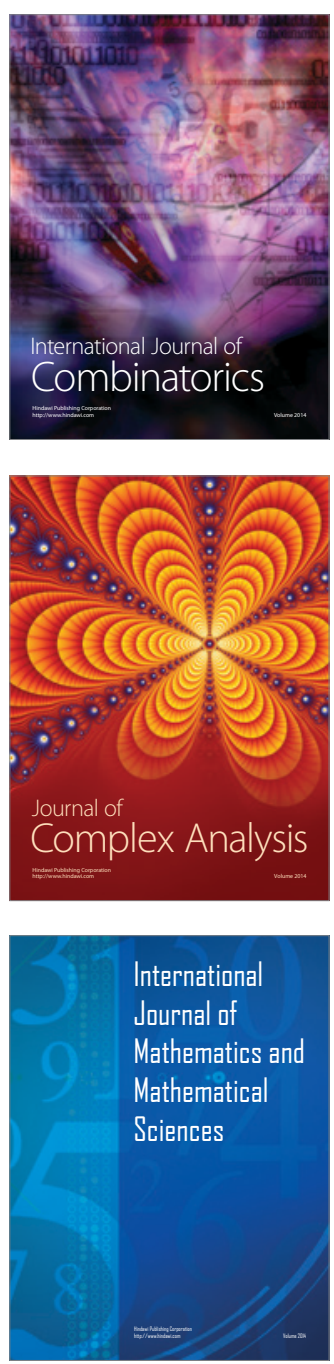
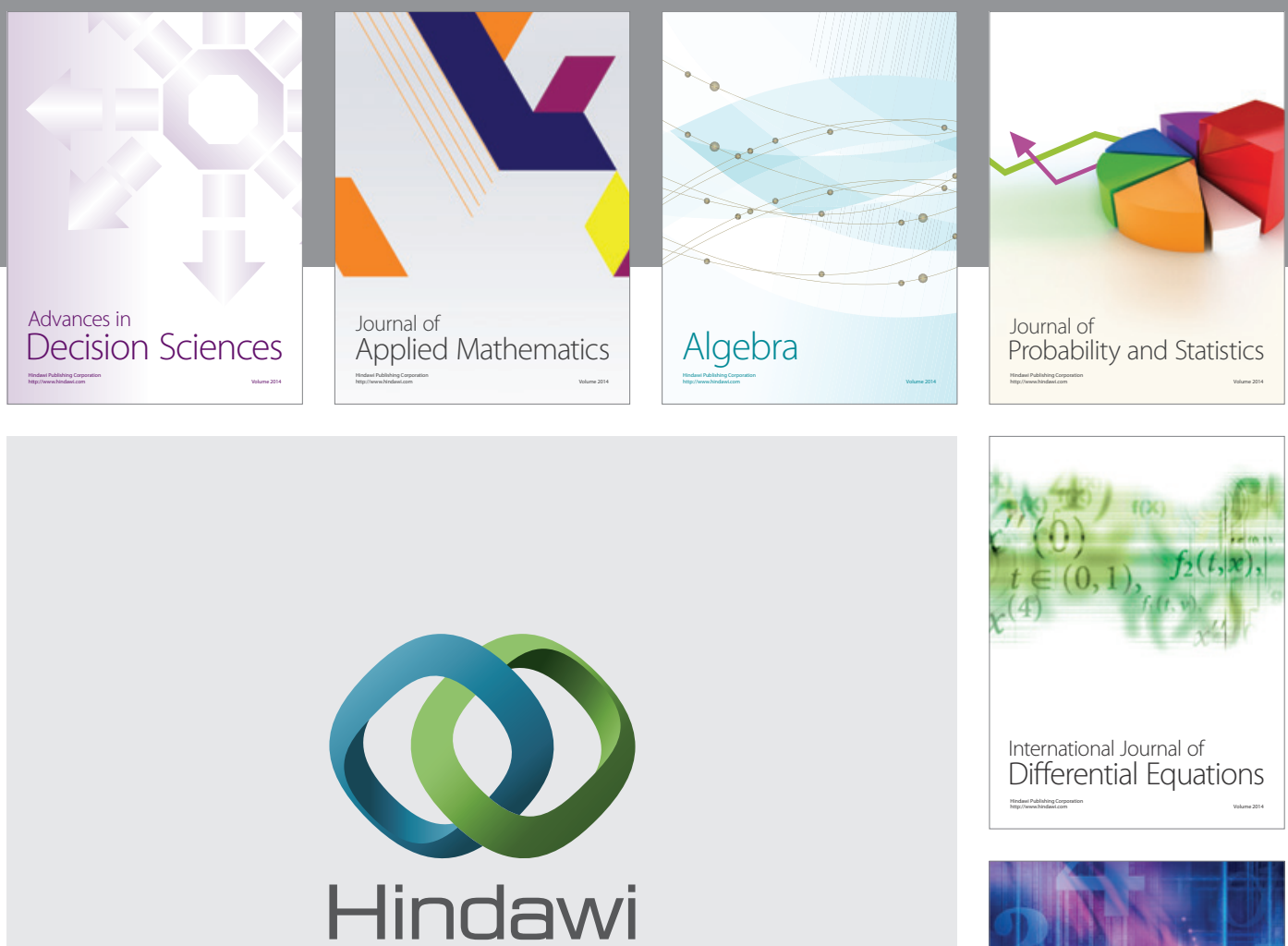

Submit your manuscripts at http://www.hindawi.com
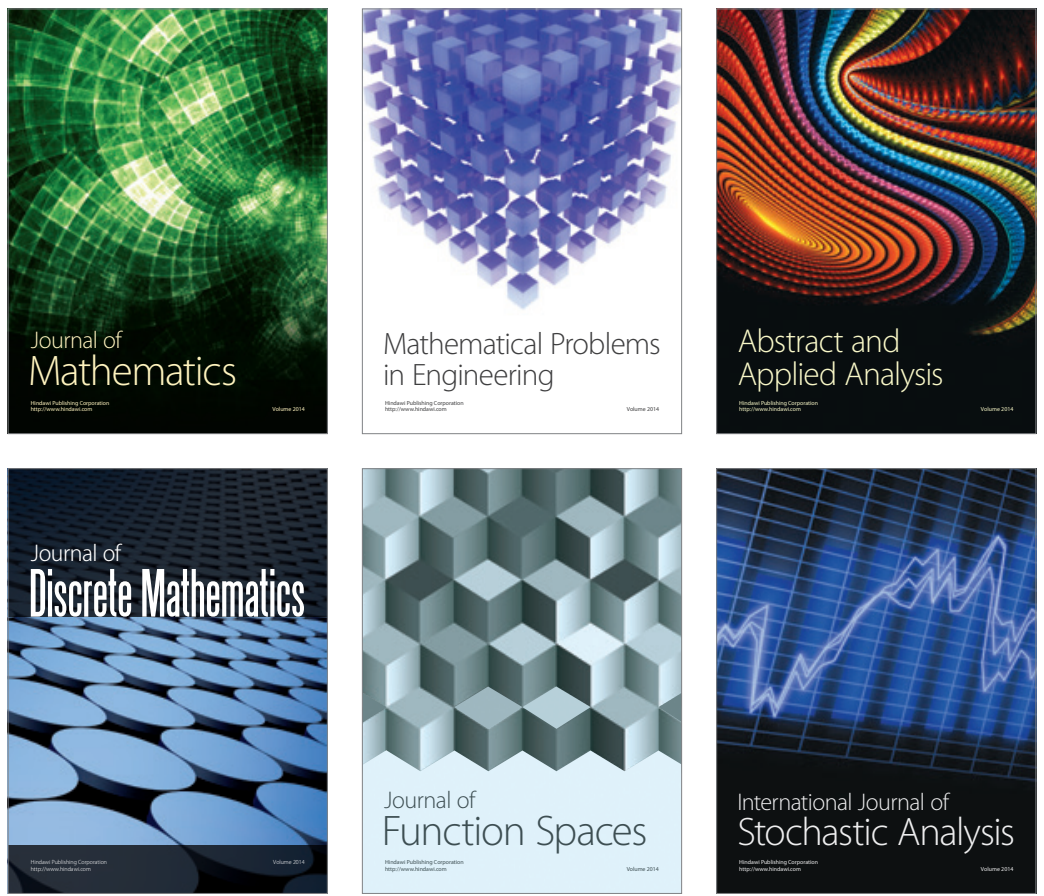

Journal of

Function Spaces

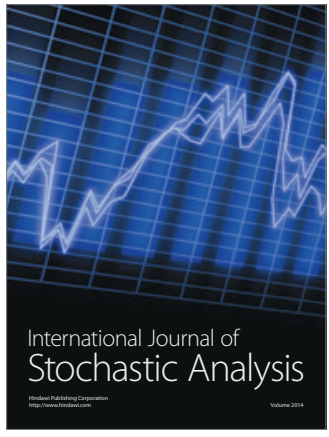

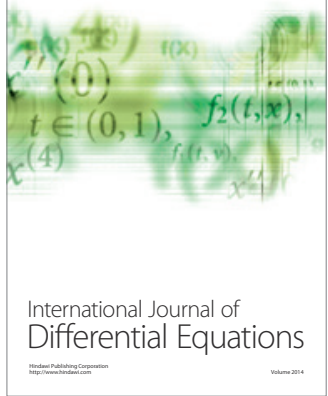
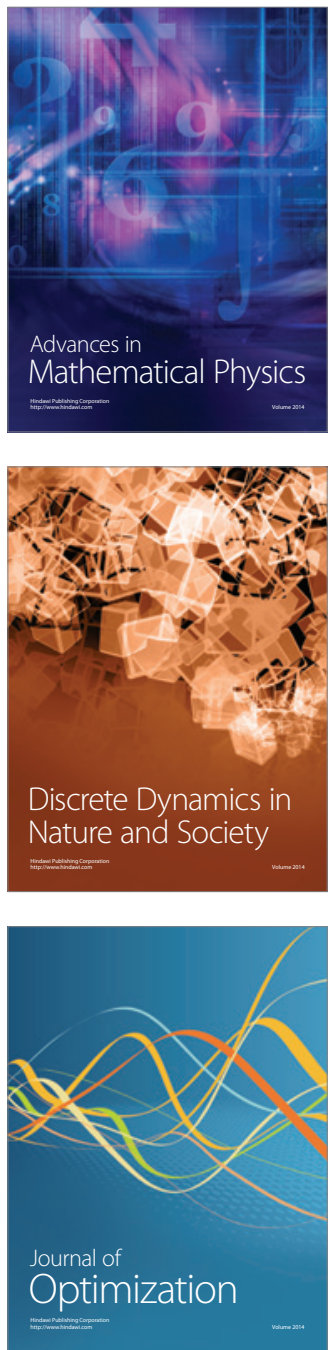